\title{
Risks of on-pump coronary artery bypass grafting surgery in patients with chronic obstructive pulmonary disease due to sulfur mustard
}

\author{
Mehdi Dehghani Firoozabadi ${ }^{1}$, Mohammad Ali Sheikhi' ${ }^{2}$, Hossein Rahmani ${ }^{3}$, Ahmad Ebadi ${ }^{4}$, Amanollah Heidari $^{5}$, \\ Behnam Gholizadeh ${ }^{5}$, Khosrow Sharifi ${ }^{5}$
}

\begin{abstract}
${ }^{1}$ Department of Anesthesiology, Tehran Heart Center, Tehran University of Medical Sciences, Tehran, Iran
2Department of Cardiac Surgery, Atherosclerosis Research Center Golestan Hospital, Ahvaz Jundishapur University of Medical Sciences, Ahvaz, Iran

${ }^{3}$ Norman Bethune College of Medicine, Jilin University, Changchun, China

${ }^{4}$ Department of Cardiac Anesthesiology, Atherosclerosis Research Center Golestan Hospital, and Pain Research Center, Ahvaz Jundishapur University of Medical Sciences, Ahvaz, Iran

${ }^{5}$ Department of Cardiac Surgery, Atherosclerosis Research Center and Imam Hospital, Ahvaz Jundishapur University of Medical Sciences, Ahvaz, Iran
\end{abstract}

Adv Dermatol Allergol 2017; XXXIV (5): 429-432

DOI: https://doi.org/10.5114/ada.2017.71107

\begin{abstract}
Sulfur mustard (SM) is a toxic chemical agent that belongs to a class of vesicant compounds. In the 1980s it was used by the Iraqi army against Iranian forces. Sulfur mustard severely irritates the skin, eyes and lungs. The highest side effects seen in patients affected by this gas are pulmonary complications including different types of lung diseases such as bronchiolitis. It has also led to a certain type of chronic obstructive pulmonary disease called mustard lung. Similar extra-pulmonary, molecular and hormonal effects can be observed in these patients and patients with chronic obstructive pulmonary disease. Here cardiovascular complications may be one of the most dangerous visible effects. And atherosclerosis is probable following the direct effects or consequential long-term effects of SM. The development of atherosclerosis in these patients is associated with an increased risk of cardiovascular and coronary artery disease. Coronary artery bypass grafting surgery is the treatment of coronary artery disease. Doing this surgery by bypass pump has its own morbidity and due to local and systemic inflammation changes in patients with SM pulmonary disorders it may have more side effects. Therefore, detailed knowledge of inflammatory diseases as well as the serum level or even the local lung fluid of the inflammatory factors in these patients before surgery are needed so that it would be possible to reduce the rate of morbidity and mortality by normalizing the inflammatory conditions of the patients before cardiac surgery.
\end{abstract}

Key words: sulfur mustard, chronic obstructive pulmonary disease, coronary artery bypass grafting, inflammatory response, mustard lung.

\section{A brief history of sulfur mustard}

Sulfur mustard (SM) or bis (chloroethyl) sulfide with a molecular formula of $\mathrm{C}_{4} \mathrm{H}_{8} \mathrm{Cl}_{2} \mathrm{~S}$ is a toxic chemical agent that belongs to a class of vesicant compounds [1, 2]. César-Mansuète Despretz and Alfred Riche reported SM synthesis from the ethylene and sulfur dioxide reaction in 1822 and 1854 [3]. This gas was first used during World War I, and restricted amounts were used in other cases, but in 1980s it was used by the Iraqi army against Iranian forces [4]. Sulfur mustard is severely irritating to the skin, eyes and lungs [5] and makes these tissues susceptible to attack and damage and causes dangerous side effects in both acute and chronic phases [6]. Despite significant studies, unfortunately no specific antidote has been introduced for SM damage [7]. The highest side effects seen in patients affected by this gas are pulmonary complications including different types of lung diseases such as bronchiolitis $[8,9]$. It has also led to a certain type of chronic obstructive pulmonary disease called mustard lung $(\mathrm{ML})$ that is not only associated with local, but

Address for correspondence: Mohammad Ali Sheikhi, Department of Cardiac Surgery, Atherosclerosis Research Center Golestan Hospital, Ahvaz Jundishapur University of Medical Sciences, 123456 Ahvaz, Iran, phone: +98 9168013916,

e-mail: mohammadalisheikhi2016@gmail.com

Received: 21.10.2016, accepted: 31.10.2016. 
also brings systemic complications [10]. Obviously the extra-pulmonary effects in these patients lead to other diseases as well. Perhaps chronic obstructive pulmonary disease (COPD) patients are the closest patients to those who suffer from ML, which of course have different etiologies [11, 12]. But similar extra-pulmonary, molecular and hormonal effects can be observed in these patients $[13,14]$. Meanwhile the cardiovascular complications may be one of the most dangerous visible effects.

\section{Atherosclerosis in patients with pulmonary complications exposed to sulfur mustard}

In the Iranian population, cardiovascular and coronary artery disease is one of the major reasons of morbidity and mortality. It is now the first cause of death in people aged about 40 . The cardiovascular disease mortality rate has been reported to be about $28 \%$ to $48 \%$, and the prevalence of ischemic heart has been reported to be rising $[15,16]$. Atherosclerosis is one of inflammatory diseases. Atherosclerosis increases due to a lot of causes such as hyperlipidemia, hypertension, diabetes mellitus, metabolic syndrome, smoking, obesity, sedentary lifestyle, and excessive alcohol consumption [17, 18].

Scientists have considered an atherosclerotic plaque gap as the clot formation initiator. Later, researchers found that the removal of the endothelial layer on the atherosclerotic plaque can activate this process [19, 20]. Now it has become clear that clot formation on the ruptured atherosclerotic plaque or a plaque the endothelium layer of which is removed is the cause of acute myocardial infarction, unstable angina and sudden death in many cases that are known as acute coronary syndromes [21, 22].

In addition to all the listed items for atherosclerosis, this complication occurs in chemical lung patients and along with other SM complications, and is considered as one of the riskiest complications. In fact, SM as a toxic factor after inhaling and in its chronic phase leads to atherosclerosis and cardiovascular and coronary artery disease [23-25].

The review of the conducted studies shows a significant incidence of cardiovascular and coronary artery disease in these patients. For example, in the study conducted by Shabestari et al. [24] , it is mentioned that among the 40 poisoned patients studied, 15 (37.5\%) had coronary artery ectasia, mainly in the left anterior descending artery, but 25 (62.5\%) did not. These values were 2 (5\%) and 38 (95\%), respectively, in the non-exposed group, which was significantly different compared to the exposed group ( $p=0.001)$. This study has also shown that in general the prevalence of CAE in mustard-poisoned patients was revealed to be 7.5 times higher than in the non-exposed controls and mustard gas is suggested to be strongly correlated with CAE in this study.

Also in another study conducted by Karbasi-Afshar et al. [26] it has been shown that 92 (92\%) patients in the SM exposed group and 82 (82\%) in the unexposed group had abnormal findings in their coronary arteries and the incidence of coronary artery disease (CAD) and angiographic changes were significantly increased with exposure to SM. Further studies on cardiovascular effects of SM are needed.

Also Gholamrezanezhad et al. [27] in another study stated that the prevalence of non-homogeneity of uptake, and left and right ventricular enlargement in both visual and quantitative analyses were higher in the mustard-exposed patients than in unexposed controls. The prevalence of ischemia was higher in the exposed patients $(p<0.05)$. The cavity to myocardium ratio, as an established and validated measure of the ejection fraction, was also significantly lower in the warfare patients than the controls. And finally the study concluded that the pattern of myocardial perfusion in these patients is significantly different from normal controls, which could resemble either coronary artery disease or mild cardiomyopathic changes.

There will be such studies in the future that indicate the prevalence of cardiovascular disease in pulmonary chemical patients exposed to SM. Therefore, paying attention to the cardiovascular complications in patients along with other complications is a key point in maintaining the health of these patients.

\section{Possible coronary artery bypass grafting complications in pulmonary chemical patients exposed to sulfur mustard}

One of the most appropriate treatment options in patients with CAD is coronary artery bypass grafting (CABG) $[28,29]$. The candidate patients for this surgery are examined in terms of various risk factors before the surgery and of course the prognosis of patients plays a key role in managing patients during and after the surgery [30, 31]. The patient's age, gender, comorbidities, the level of coronary artery stenosis, and ejection fraction are important items in the prognosis of the patient's condition [32]. In addition to important mortality and morbidities in this surgery, one of the major problems is the systemic changes following the use of the bypass pump [33]. This complication can be more severe in patients exposed to SM because the SM alone or the cellular and molecular mechanisms caused by it lead to changes in the patient's systemic range [34,35] and now the use of the bypass pump in these patients adds to the severity of the systemic effects.

The use of cardiopulmonary bypass (CPB) in order to enable open heart surgery is among the most important clinical advances in medicine in the twenty first century and has led to more developments such as external respiratory support, mechanical circulatory support, ventricular assist devices and artificial hearts [36, 37]. The method is used in today's world by heart and thoracic 
surgeons. In addition to the significant benefits of this device, there are many complications associated with this system the most important of which are systemic inflammation and severe changes in the inflammatory system of the patient [38]. The CPB circuit has an interface with blood flow which is not at all similar to normal endothelium and this causes adverse reactions. Activation of the complement system is the primary mechanism that initiates and reinforces the acute inflammatory response. In the inactivated mode, the component system consists of nine components with the digits C1-C9. Also changes occur in various inflammatory factors including interleukins and acute phase protein reactions. All these changes lead to secondary systemic and even diseases in the blood, liver and kidneys [39, 40].

Sulfur mustard pulmonary patients suffer from severe inflammatory changes following the long-term SM complications and it is observed that the inflammatory markers such as interleukins, matrix metalloproteinases have had lasting changes in these patients [41, 42]. Now the exposure of these patients with a bypass pump and inflammatory complications could lead to a storm of inflammatory reactions and subsequent systemic and local severe complications. Therefore, special care of the patient before the surgery and the possibility of inflammatory local and even systemic pulmonary changes is a golden point in the management of these patients.

\section{Management of candidate sulfur mustard pulmonary patients for coronary artery bypass grafting}

As mentioned before, the SM pulmonary patients are subject to morbidity and mortality caused by CABG surgery more than other people because along with other existing risk factors such as changes in pulmonary volumes and capacities, renal diseases or other disorders, they suffer from severe inflammatory changes and this complication in the patient with cardiac surgery with a bypass pump can be a dangerous threshold of systemic inflammation changes [43-45]. It is observed that different interleukins in these patients have had an increased or decreased serum level and also unlike most studies that have shown upward changes in these patients, the markers decreased in some patients despite a history of cancer [46-48]. It is also observed that the bypass pump can cause changes in creatinine and subsequent renal disease in patients [49], while disturbing the trend of inflammation markers in SM pulmonary patients, and adds to the development of this complication and this evidence indicates the lack of transparency of the SM mechanism in the human body and its immune system. Therefore, detailed knowledge of inflammatory diseases as well as the serum level and even localizing lung fluid of the inflammatory factors in these patients is needed before surgery so that by normalizing the inflammatory condition of the patient before cardiac surgery, it would be possible to reduce the possible morbidity and mortality.

\section{Conflict of interest}

The authors declare no conflict of interest.

\section{References}

1. Vučemilović A, Hadžija M, Jukić I. Efficacy of mineral cationic carrier against sulphur mustard in skin decontamination. Arch Indust Hyg Toxicol 2008; 59: 289-93.

2. Rahmani H, Javadi I, Shirali S. Respiratory complications due to sulfur mustard exposure. Int J Curr Res Acad Rev 2016; 4: 143-9.

3. Etemad L, Moshiri M, Balali-Mood M. History of use and epidemiology of mustard compounds. In: Basic and Clinical Toxicology of Mustard Compounds. Balali-Mood M, Abdollahi M (eds.). Springer International Publishing 2015; 29-47.

4. Mansour Razavi S, Salamati P, Saghafinia M, Abdollahi M. A review on delayed toxic effects of sulfur mustard in Iranian veterans. DARU J Pharm Sci 2012; 20: 51.

5. Tahmasbpour E, Ghanei M, Qazvini A, et al. Gene expression profile of oxidative stress and antioxidant defense in lung tissue of patients exposed to sulfur mustard. Mutation Res Genet Toxicol Environ Mutagen 2016; 800: 12-21.

6. Balali-Mood M, Hefazi M. The pharmacology, toxicology, and medical treatment of sulphur mustard poisoning. Fund Clin Pharmacol 2005; 19: 297-315.

7. Weber WM, Kracko DA, Lehman MR, et al. Inhalation exposure systems for the development of rodent models of sulfur mustard-induced pulmonary injury. Toxicol Mechanisms Methods 2010; 20: 14-24.

8. Panahi Y, Ghanei M, Vahedi E, et al. Efficacy of probiotic supplementation on quality of life and pulmonary symptoms due to sulfur mustard exposure: a randomized double-blind placebo-controlled trial. Drug Chem Toxicol 2017; 40: 24-9.

9. Akbarzadeh A, Abasi E, Ghanei M, et al. The effects of various chemicals on lung, skin and eye: a review. Toxin Rev 2016; 35: 187-95.

10. Lari SM, Attaran D, Towhidi M. COPD Due to Sulfur Mustard (Mustard Lung). INTECH Open Access Publisher 2012.

11. Sahebkar A, Antonelli-Incalzi R, Panahi Y, et al. Mustard lung and COPD: common features and treatment? Lancet Respir Med 2015; 3: 747-8.

12. Shahriary A, Seyedzadeh MH, Ahmadi A, Salimian J. The footprint of TGF-beta in airway remodeling of the mustard lung. Inhalat Toxicol 2015; 27: 745-53.

13. Hassan ZM, Ebtekar M. Immunological consequence of sulfur mustard exposure. Immunol Letters 2002; 83: 151-2.

14. Weinberger B, Laskin JD, Sunil VR, et al. Sulfur mustard-induced pulmonary injury: therapeutic approaches to mitigating toxicity. Pulmon Pharmacol Therap 2011; 24: 92-9.

15. Ali SM, Ebadi A, Rahmani H. Importance of further recognition of atherosclerotic patients candidate for CABG: predisposing factors for postoperative stroke. Adv Nat Appl Sci 2015; 9: 8-13.

16. Firoozabadi MD, Ebadi A, Ebadi M, et al. Prevalence risk factors in three vessel disease patients candidate for coronaryartery bypass surgery. Life Sci I 2014; 11: 67-70.

17. Firoozabadi MD, Ebadi A, Sheikhi MA, Rahmani H. Effect of general anesthesia plus spinal anesthesia on patients hemodynamic during coronary artery bypass grafting surgery. Der Pharma Chem 2016; 8: 301-5. 
18. Sheikhi MA, Ebadi A, Ramezani A, Gholizadeh B. From atherosclerosis to CABG. Int J Bioass 2015; 4: 3676-81.

19. Libby P, Theroux P. Pathophysiology of coronary artery disease. Circulation 2005; 111: 3481-8.

20. Heusch G, Libby P, Gersh B, et al. Cardiovascular remodelling in coronary artery disease and heart failure. Lancet 2014 383: 1933-43.

21. Falk E, Nakano M, Bentzon JF, et al. Update on acute coronary syndromes: the pathologists' view. Eur Heart J 2013; 34: 719-28

22. Eggers KM. Defining acute myocardial infarction. Heart Metab 2015; 67: 34-8.

23. Namazi S, Niknahad H, Razmkhah H. Long-term complications of sulphur mustard poisoning in intoxicated Iranian veterans. J Med Toxicol 2009; 5: 191-5.

24. Shabestari MM, Jabbari F, Gohari B, et al. Coronary artery angiographic changes in veterans poisoned by mustard gas. Cardiology 2011; 119: 208-13.

25. Rohani A, Akbari V, Moghadam FT. A case control study of cardiovascular health in chemical war disabled Iranian victims. Indian J Crit Care Med 2010; 14: 109-12.

26. Karbasi-Afshar R, Shahmari A, Madadi M, et al. Coronary angiography findings in lung injured patients with sulfur mustard compared to a control group. Ann Cardiac Anaesth 2013; 16: 188-92.

27. Gholamrezanezhad A, Saghari M, Vakili A, et al. Myocardial perfusion abnormalities in chemical warfare patients intoxicated with mustard gas. Int J Cardiovasc Imaging 2007; 23: 197-205.

28. Sheikhi MA, Ebadi A, Rahmani H. Importance of further recognition of atherosclerotic patients candidate for CABG: predisposing factors for postoperative stroke. Adv Nat App Sci 2015; 9: 8-13.

29. Sheikhi MA, Ebadi A, Gholizadeh B, Ramezani A. Risk factors for blood pressure change after CABG surgery in southwest of Iran. Int J Bioassay 2015; 4: 3659-63.

30. Restrepo L, Wityk RJ, Grega MA, et al. Diffusion-and perfusion-weighted magnetic resonance imaging of the brain before and after coronary artery bypass grafting surgery. Stroke 2002; 33: 2909-15.

31. Ebadi A, Soltanzadeh M, Nesioonpour S, et al. Effects of angiotensin converting enzyme inhibitors before, during and after coronary artery bypass graft surgery on hemodynamic responses and vasoactive drugs requirement. Anesth Pain Med 2014; 4: e16510.

32. Diegeler A, Börgermann J, Kappert U, et al. Off-pump versus on-pump coronary-artery bypass grafting in elderly patients. N Engl J Med 2013; 368: 1189-98.

33. Jongman RM, Zijlstra JG, Kok WF, et al. Off-pump CABG surgery reduces systemic inflammation compared with onpump surgery but does not change systemic endothelial responses: a prospective randomized study. Shock 2014; 42: 121-8.

34. Riahi-Zanjani B, Mahmoudi M. Immunological and hematological complications of sulfur mustard poisoning. In: $\mathrm{Ba}$ sic and Clinical Toxicology of Mustard Compounds. BalaliMood M, Abdollahi M (eds.). Springer International Publishing 2015; 273-89.

35. Ghanei M, Harandi AA. Molecular and cellular mechanism of lung injuries due to exposure to sulfur mustard: a review. Inhal Toxicol 2011; 23: 363-71.

36. Apostolakis E, Filos KS, Koletsis E, Dougenis D. Lung dysfunction following cardiopulmonary bypass. J Card Surg 2010; 25: 47-55.
37. Murphy GS, Hessel EA, Groom RC. Optimal perfusion during cardiopulmonary bypass: an evidence-based approach. Anesth Analg 2009; 108: 1394-417.

38. Abu-Omar Y, Ratnatunga C. Cardiopulmonary bypass and renal injury. Perfusion 2006; 21: 209-13.

39. Ebadi MA, Shahriary A, Davoodzadeh H, Rahmani H. Cardiac surgery anesthesia and systemic inflammatory response. Int J Bioassays 2015; 4: 3648-55.

40. Asimakopoulos G. The inflammatory response to CPB: the role of leukocyte filtration. Perfusion 2002; 17 (2 Suppl): 7-10.

41. Keramati MR, Balali-Mood M, Mousavi SR, et al. Biochemical and hematological findings of Khorasan veterans 23 years after sulfur mustard exposure. I Res Med Sci 2013; 18: 855-9.

42. Kiani A, Mostafaie A, Shirazi FH, Ghazanfari T. Serum profiles of matrix metalloproteinases and their tissue inhibitors in long-term pulmonary complication induced by sulfur mustard: Sardasht-Iran Cohort Study (SICS). Int Immunopharmacol 2013; 17: 964-7.

43. Balali-Mood M, Balali-Mood B. Sulphur mustard poisoning and its complications in Iranian veterans. Iran J Med Sci 2015; 34: 155-71.

44. Pourfarzam S, Ghazanfari T, Yaraee R, et al. Serum levels of IL-8 and IL- 6 in the long term pulmonary complications induced by sulfur mustard: Sardasht-Iran Cohort Study. Int Immunopharmacol 2009; 9: 1482-8.

45. Panahi Y, Jadidi-Niaragh F, Azimzadeh Jamalkandi S, et al. Immunology of chronic obstructive pulmonary disease and sulfur mustard induced airway injuries: implications for immunotherapeutic interventions. Curr Pharm Des 2016; 22: 2975-96.

46. Yaraee R, Ghazanfari T, Ebtekar M, et al. Alterations in serum levels of inflammatory cytokines (TNF, IL-1alpha, IL-1beta and IL-1Ra) 20 years after sulfur mustard exposure: Sardasht-Iran cohort study. Int Immunopharmacol 2009; 9: 1466-70.

47. Sheikhi MA, Rahmani H. Inflammatory statuses of nonsmoker mustard lung patient candidate for coronary artery bypass grafting surgery. Int I Pharm Res Allied Sci 2016; 5: 194-5.

48. Heidari A, Sheikhi MA, Rahmani H. Inflammatory status of non-smoker sulphur mustard exposed patient with cancer candidate for coronary artery bypass grafting surgery. Int J Pharm Res Allied Sci 2016; 5: 196-8.

49. Firoozabadi MD, Ebadi A. The effect of oral $\mathrm{N}$-acetylcysteine on serum creatinine in chronic kidney diseases patients under CABG surgery. Life Sci J 2014; 11: 138-43. 